\title{
LARGE SCALE JETS IN CLASS I AND CLASS II RADIO SOURCES AND QUASARS
}

\author{
G.V. BICKNELL \\ Mount Stromlo and Siding Spring Observatories \\ Institute of Advanced Studies \\ Australian National University
}

\begin{abstract}
The physics of large scale jets in class I and class II extragalactic radio sources and quasars is discussed. Class I jets appear to be turbulent, transonic jets which entrain the interstellar medium. The related jet deceleration causes a slow surface brightness decline which is usually observed. Class II jets are supersonic and terminate in an advancing shock against the external medium. Both types of jet are initially light but the ratio of jet density to external density of class $I$ jets increases owing to entrainment. It is quite plausible that quasar jets are hypersonic and light and this may solve problems of confinement. The velocities of class $I$ jets are of the order of a few thousand kilometers per second. Class II and quasar jets may be at least mildly relativistic. However, it is not clear whether the velocities of large scale jets in powerful sources are close to the speed of light. Recent depolarization measurements provide an interesting focus for discussion of this question.
\end{abstract}

\section{Introduction}

Almost a decade and a half ago Fanaroff and Riley (1974) demonstrated a distinct morphological difference between radio sources whose total powers are respectively less than and greater than the critical value

$$
P_{\text {tot }}^{1.4 \mathrm{GHz}} \approx 10^{24.5} \mathrm{~W} \mathrm{~Hz}^{-1}
$$

They noted that the low luminosity (class I) sources are edge-darkened whereas the high luminosity (class II) sources have their brightest emitting regions towards their extremities. This morphological difference is illustrated in figure 1 which is a VLA radio image of the radio source in the cluster Abell 3744 (Cameron, 1988). Fortuitously, this cluster contains an example of both generic types (albeit with large scale structure which is influenced by the properties of the intracluster medium and the velocities of the galaxies with respect to it).

Fanaroff and Riley noted that the morphological difference between class I and class II sources probably reflects the different manner in which energy is transported in the two types of source. At around the same time, Blandford and Rees (1974) and Scheuer (1974) 
Figure 1. An N.R.A.O. V.L.A. image of the radio source PKS 2104-25 in the cluster Abell 3744. The class I source is the southern component and is associated with the elliptical galaxy NGC 7016; the class II source is the northern component and is associated with the dumbell galaxy NGC 7018. The N.R.A.O is operated by Associated Universities Inc. under contract to the National Science Foundation.

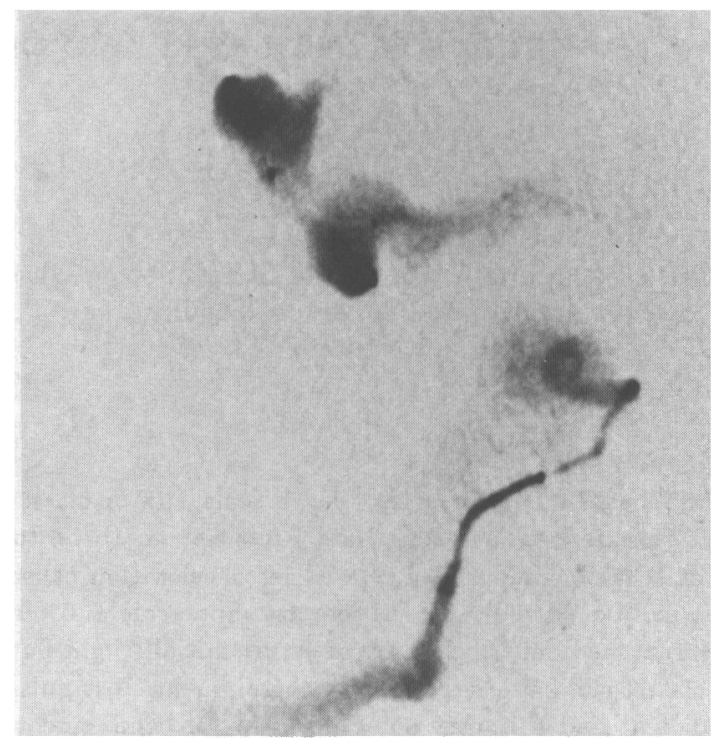

published papers proposing that "beams", consisting of energetic particles and magnetic field transported the energy to the lobes of radio sources over a timescale $\sim 10^{8} \mathrm{yrs}$. The subsequent detection of "jets" between the core and extended lobes of radio sources verfied this idea and it became apparent from later work that the major differences between class I and class II sources are strongly related to the physics of jet propagation, bearing out Fanaroff's and Riley's original proposition. For instance, Bridle (1984) showed that there are strong systematic differences between jets in class I and class II ssources. In particular, class I sources tend to have two jets of similar brightness whereas class II sources are generally one-sided with respect to their jets. Thus, over the last few years, the study of radio sources has been dominated by the study of the jets they contain. The reward, for what has been an intensive observational and theoretical effort which can not be covered adequately in this short review, is that the relevant physical differences between the two types of radio source are beginning to be understood. Moreover, the detailed study of jets in quasars (see, for example Barthel et al 1988a,b) has added a fascinating extra dimension to the studies of jets. However, despite the progress that has been made in this field, the question of the velocities of jets, in particular class II jets, remains a controversial one. The recent discovery of substantial depolarization variations in powerful radio sources has added significant fuel to the debate and I shall come to this topic towards the end of this paper. The first topics I shall discuss relate to the physics which is considered to be relevant for class I, class II and quasar jets and the models which have been constructed to explain them. 


\section{Class II Jets}

The first models that were constructed for radio sources were directed towards understanding the properties of powerful radio sources. Thus it is appropriate to discuss these first.

The paper by Blandford and Rees (1974) essentially mapped out a great deal of the physics which is relevant to the large scale structure of class II radio sources. In a class II source a supersonic jet terminates at a "hotspot" via a shock against the intergalactic or interstellar medium. A bow shock precedes the jet as it propagates out into the external medium and a contact discontinuity between the jet and external plasma is also established. The hotspot progresses outwards with a velocity $v_{\mathrm{h}} \approx \eta^{-1 / 2} v_{\text {jet }}$ where the important parameter $\eta$ is the ratio of the jet density to the density of the surrounding medium. Most lobes in class II radio galaxies are observed to be large compared to the related jets, when they can be detected, and the parameter $\eta$ has the largest effect on this. The expansion of the lobe is the greater the smaller $\eta$ is and the axisymmetric simulations by Norman et al. (1984) demonstrate this effect quite clearly. This raises the possibility that $\eta$ could be estimated from the relative size of the jet and the lobe. However, wiggling of the end of the jet can lead to a much larger lobe and the production of multiple hot spots (Scheuer 1974). This has been verified by the three dimensional simulations of Williams and Gull (1984) and recent higher resolution simulations of two dimensional slab jets by Norman and Hardee (1988) (see figure 2). Nevertheless, jet wiggling can not account for the entire size of a lobe and at present most numerical modeling of class II sources entails values of $\eta<0.01-0.1$. This is also probably the correct physical range for this parameter; the lowest realistic limit to $\eta$ is unknown at present.

Figure 2. Simulation of a supersonic slab jet by Norman and Hardee (1988). Oscillation of the end of the jet causes it to generate a larger cavity than would be the case if the jet were perfectly straight.

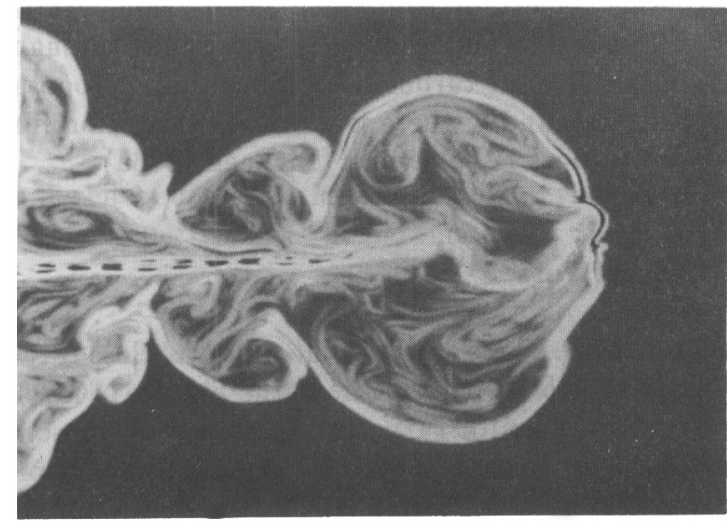

With the high resolution provided by the V.L.A. and Merlin interferometers, radio astronomers have been able to observe more detail in distant sources. In particular, quasars have revealed intetesting jet structure (see, for example Potash and Wardle 1979, Barthel et al. 1988a,b) which poses some important questions. Jet confinement often proves to be a problem enunciated clearly by Pottash and Wardle. Moreover, while a number of quasars have typical class II morphology, many are bent or distorted indicating significant 
interaction with large $\left(\sim 10^{7}-10^{8} M_{\odot}\right)$ clouds in the ambient medium. Some quasar jets, e.g. 3C273 (Davis et al. 1985), do not have the typical class II morphology, lacking the usual inflated lobe at the end of the jet. One possibility therefore, is that these jets are heavy but this would be surpising in view of what appears to be the case in powerful class II radio galaxies. Norman (1986) has proposed an attractive alternative and that is that these jets are light and hypersonic. In this flow regime the bow shock is highly swept back preventing the lateral expansion of shocked jet plasma. Moreover, the pressure behind the advancing bow shock is well above ambient and confines the jet. Thus Norman's model provides a neat solution for both the morphology and the apparent overpressure of quasar jets. The idea of a hypersonic jet also fits in qualitatively with the generally higher quasar power output.

One of the early objections to beam models of radio sources was that beams would be Kelvin-Helmholtz unstable. Supersonic, light jets tend to avoid destructive KelvinHelholtz effects through the "reflecting mode" which sets in when

$$
v_{\text {jet }}>c_{\mathrm{s}, \text { int }}+c_{\mathrm{s}, \mathrm{ext}}
$$

where $c_{\mathrm{s}, \text { int }}$ and $c_{s, \text { ext }}$ are the internal and external jet sound speeds (Payne and Cohn 1985). This mode is related to internal waves which are reflected from the surface of the jet and which saturate in travelling biconical shocks (Norman et al. 1984). These shocks are less disruptive than the stationary "ordinary mode" shocks which can occur in lower velocity, higher density radio jets. Reflecting mode shocks may be responsible for at least some knots observed in class II jets.

Another aspect of jets which saves them from destruction by the Kelvin Helmholtz instability is the fact that they propagate through a pressure gradient which tends to reduce the level of turbulence. It has been proposed that this results in a critical Mach number of around 2 where jets make a transition from class I behaviour (see the following section) to class II behaviour (Bicknell 1986a).

\section{Class I Jets}

Whereas the physics of jets in the powerful class II radio sources is dominated by the physics of supersonic or hypersonic flow, the physics of low luminosity class I sources is dominated by considerations of turbulent transonic or subsonic flow. There are two main reasons for this: Near their respective cores, class I jets spread more rapidly than can usually be accounted for by adiabatic laminar flow in a normal galaxy atmosphere and they decline in surface brightness more slowly than expected for a constant velocity laminar jet. It is natural to interpret the spreading as being due to turbulent expansion and the second property as being due to the compression of matter and fields by the related entrainment (Fanti et al. 1982, Bicknell 1984, 1986b). Models of class I jets have generally focussed on possible explanations for the surface brightness behaviour since this was judged as the main feature of class I jets that was most difficult to understand. Nevertheless, the realistic calculation of turbulent flow models is fraught with a number of difficulties, not the least 
of which is the turbulent closure problem for light, transonic flow. This is highlighted by the following ensemble-averaged equation for the $z$-momentum of a jet:

$$
\frac{\partial}{\partial z}\left(\bar{\rho} \tilde{v}_{z}^{2}\right)+\frac{1}{r} \frac{\partial}{\partial r}\left(r \bar{\rho} \tilde{v}_{r} \tilde{v}_{z}\right)=-\left(1-\frac{\bar{\rho}}{\rho_{\mathrm{ext}}}\right) \frac{d P_{\mathrm{ext}}}{d z}-\frac{1}{r} \frac{\partial}{\partial r}\left(r\left\langle\rho v_{r}^{\prime} \tilde{v}_{z}^{\prime}\right\rangle\right)
$$

In order to solve this, and the related hydrodynamic equations, it is necessary to relate the Reynolds stress, $\left\langle\rho v_{r}^{\prime} \tilde{v}_{z}^{\prime}\right\rangle$, to the variables describing the mean flow. Unfortunately, such a prescription will probably never become available for light transonic/supersonic flow and detailed direct modelling of such flows (necessarily three dimensional and of high resolution) requires at least the capabilities of modern supercomputers. It is possible to make useful progress however, using a semi-empirical approach (Bicknell 1986b) wherein data on the spreading rate of class I jets can be used to infer the rate of change of velocity and surface brightness.

As is evident from the above equation the local external pressure gradient is an important input to these models. A simple isothermal atmosphere is used whose pressure is described by:

$$
\frac{P}{P_{c}}=\exp \left[-\beta \frac{\left(\phi-\phi_{c}\right)}{\sigma^{2}}\right]
$$

where the c subscripts indicate central values and $\phi$ is a King potential with velocity dispersion $\sigma$. This has many of the features of a more realistic atmosphere model, namely a power law region outside the core followed by a flattening to a background pressure at large radii. The crucial parameter in these models is $\beta$, the ratio of the virial temperature to the ISM temperature.

The surface brightness of a jet described by such an adiabatic model goes as

$$
I_{\nu} \propto\left(\tilde{v}_{z} a^{2}\right)^{-s / 3} \times\left(\tilde{v}_{z} a\right)^{-(s-1) / 2}
$$

where the two separate factors indicate the effect of adiabatic compression of the relativistic electron distribution and the magnetic field respectively. (The parameter $s$ is the exponent of the distribution function.)

This model has been fitted to the data on a number of sources. Most recently, Morganti et al. (1988) have fit the surface brightness data on 23 jets from the Bologna survey obtaining satisfactory fits in most cases. An important result is that the initial value of the parameter $\eta$, the ratio of the jet density to the interstellar medium density is less than unity, consistent with the inferred situation in class II sources. Entrainment causes this parameter to increase towards unity. The model fits also generally lead to an initial Mach number between 1 and 2, consistent with the notion that these jets are transonic. A sample model fit to the eastern jet in the radio galaxy $\mathrm{B} 20034+25$ is given in figure 3 .

Perhaps the most satisfactory aspect of the model fits is the estimated values of the parameter $\beta$. The median value is 0.7 which is identical to the median $\beta$ inferred by Thomas et al. (1985) from an independent sample of X-ray ellipticals. 
Figure 3. The FWHM data (left panel) and a light, transonic jet model fit (right panel) to the surface brightness of the eastern jet in the source B2 $0034+25$.
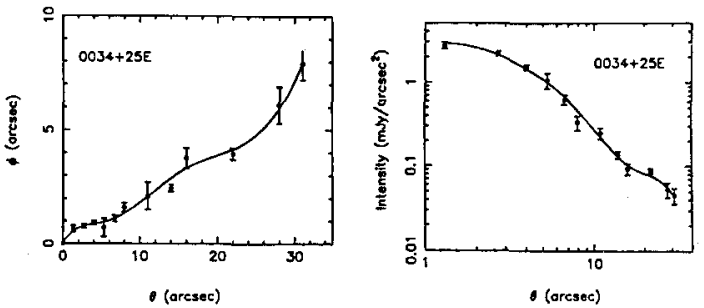

The notion of low Mach numbers for class I jets is supported by the work of O'Dea (1985) on the characteristics of bent jets in clusters of galaxies.

\section{Jet Velocities and Depolarization}

With the understanding that class I jets are low Mach number, confined jets with $\eta \sim .01-1$, the implied velocities

$$
v_{\mathrm{jet}}=4.3 \times 10^{7} \eta^{-1 / 2} M\left(\frac{T_{\mathrm{ism}}}{10^{7} \mathrm{~K}}\right)^{1 / 2} \mathrm{~km} \mathrm{~s}^{-1}
$$

where $\mathrm{M}$ is the Mach number and $T_{\text {ism }}$ is the temperature of the interstellar medium, are of the order of a few thousand kilometers per second. Velocities of this magnitude are consistent with the energy budget in class I sources (Bicknell 1986a). Allowing that class II jets have higher Mach numbers and possibly lower density ratios, at least mildly relativistic velocities are possible. This has also been argued for by Dreher (1984) on the basis of an energy budget calculation relevant to this type of source. Nevertheless, the question of whether jet velocities are relativistic on the large scale is a controversial one at present. Relativisitic velocities have long been invoked as an explanation for the sidedness of jets in powerful sources through doppler boosting/dimming of the radiation from the approaching/receding jet. Moreover, superluminal motion, generally attributed to the effect of viewing a relativistic jet which is moving at a small angle to the line of sight, has been observed on the VLBI scale in a number of quasars (such as 3C 273, Unwin et al. 1985) and one radio galaxy, 3C 120 (Walker et al. 1987).

Recently, there have been some very interesting observations that have injected new life into the velocity debate. Laing (1988) reported results on the depolarization properties of powerful radio sources all of which have one-sided jets. From a sample of ten sources, he concluded that the jetted side is less depolarized than the non-jetted side. This conclusion was supported by Garrington et al. (1988) in a follow-up sample made up of 25 sources. One possible interpretation of this is that both jets are observed through a uniformly distributed Faraday screen which causes depolarization through differential Faraday rotation. This implies that the jetted side is closer to us, supporting the relativistic interpretation. 
Figure 4. Plot of the ratio of counter jet surface brightness to main jet surface brightness versus the ratio of spreading rates for 20 sources from the Bologna survey. In all but two cases the brighter jet spreads the least rapidly.

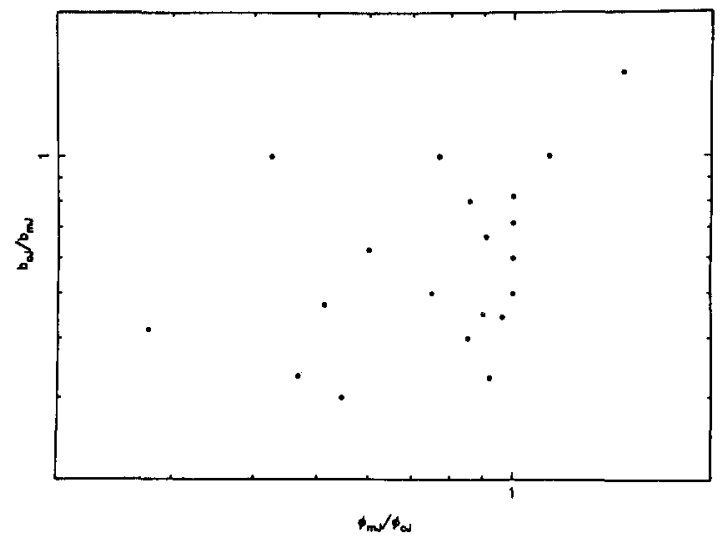

On the other hand, as both Laing and Garrington et al. remark, the depolarization asymmetry could be intrinsic, in which case one is required to search for some mechanism relating the jet asymmetry to it.

The basis for such an explanation may come from the following (Bicknell, Parma and de Ruiter 1988). Suppose we attribute the depolarization asymmetry to an asymmetric distribution of matter within the galaxy. Then it is possible that the jet propagating through the denser material is "splattered" causing it to be dimmer, partially because of less collimation. If this is the case, then one may expect to see some evidence for the effect in class I sources. Although the jet brightness ratios in class I sources are not as extreme as they are in class II sources, brightness differences do exist within the same source. Thus Parma, de Ruiter and I examined the data on a number of jets from the Bologna survey Parma et al. 1986) in order to determine whether there is any correlation between the ratio of jet surface brightnesses and the ratio of spreading rates within the same source. The result of plotting such ratios is shown in figure 4 where the ratio of the brightnesses of the counter jet and main jet at $5 \mathrm{kpc}$ is plotted against the ratio of the spreading rates. It is apparent that the fainter jet almost always (there are only two exceptions) spreads more rapidly than the brighter jet. This plot suggests that sidedness may not be related to relativistic beaming; however the final verdict is certainly not in. One phenomenon that is not immediately catered for by this splattering argument is that where a one-sided VLBI jet is observed it always points in the direction of the brighter large scale jet. If splattering is responsible for this as well, then it implies that the asymmetry in the interstellar medium extends from parsec to kiloparsec scales. Some find this unappealing. Whatever the case, the resolution of the jet sidedness question is not merely a matter of determining a number to attach to the velocity. It impacts upon the nature of jet formation and the interaction of the jet with the interstellar medium on all scales within the galaxy.

\section{Acknowledgement}

I would like to acknowledge the assistance of Paola Parma, Hans de Ruiter, Mike Norman and Phil Hardee in preparing material for this talk. 


\section{References}

Barthel P.D. and Miley G.R., 1988a, S.T.Sc.I preprint

Barthel P.D., Miley G.K., Schilizzi R.T. and Lonsdale C.T., 1988b, S.T.Sc.I preprint

Bicknell G.V. 1984, Ap.J., 239, 433

Bicknell G.V., 1986a, Ap.J., 286, 68

Bicknell G.V., 1986b, Ap.J., 305, 109

Bicknell G.V., Parma P., and de Ruiter H., 1988, in preparation

Bridle A.H., 1984, A.J., 89, 979

Blandford R.D. and Rees M.J., 1974, M.N.R.A.S., 169, 395

Cameron R.A., 1988, Ph.D. thesis, Australian National University

Davis R.J., Muxlow T.W.B. and Conway R.G., 1985, Nature, 318, 343

Dreher J., 1984, in Physics of Energy Transport in Extragalactic Radio Sources, ed. Bridle

A.H. and Eilek J.A., N.R.A.O, Greenbank, 109

Fanaroff B.L. and Riley J.M., 1974, M.N.R.A.S., 167, 31P

Fanti R., Lari C., Parma P., Bridle A.H., Ekers R.D., and Fomalont E.B., 1982, Astr. Ap., 110,69

Garrington S.T., Leahy J.P., Conway R.G., and Laing R.A. 1988, Nature, 331, 147

Laing R.A., 1988, Nature, 331, 149

Morganti R., Parma P., de Ruiter H.R., Bicknell G.V. and Fanti R., 1988, in preparation.

Norman M.L., 1986, Bull. A.A.S.

Norman M.L. and Hardee P.E., 1988, in preparation.

Norman M.L., Winkler K.-H.A., and Smarr L.L., 1984, in Physics of Energy Transport in

Extragalactic Radio Sources, ed. Bridle A.H. and Eilek J.A., N.R.A.O, Greenbank, 150

O'Dea C., 1985, Ap. J., 295, 80

Parma P., de Ruiter H.R., Fanti C., Fanti R., 1986, Astron. Astrophys. Suppl. Ser., 64, 135

Payne D.G. and Cohn H. 1985, Ap.J., 291, 655

Pottash R.I. and Wardle J.F.C., 1979, A.J., 84, 707

Scheuer P.A.G., 1974, M.N.R.A.S., 166, 513

Thomas P.A., Fabian A.C., Arnaud K.A., Forman W., Jones C., 1985, M.N.R.A.S., 222, 655

Unwin S.C., Cohen M.H., Biretta J.A., Pearson T.J., Seielstad G.A., Walker R.C., Simon R.S. and Linfield R.P., 1985, Ap.J., 289, 109

Walker R.C., Benson J.M. and Unwin S.C., 1987, Ap. J., 316, 546

Williams A.G., and Gull S.F., 1984, Nature, 310, 33 